\title{
Starspot variability and evolution from modeling Kepler photometry of active late-type stars
}

\author{
Alexander Brown ${ }^{1}$, Heidi Korhonen ${ }^{2}$, Svetlana Berdyugina ${ }^{3}$, Barton \\ Tofany $^{1}$, Thomas R. Ayres ${ }^{1}$, Adam Kowalski ${ }^{4}$, Suzanne Hawley ${ }^{4}$, \\ Graham Harper $^{5}$, and Nikolai Piskunov ${ }^{6}$ \\ ${ }^{1}$ Center for Astrophysics and Space Astronomy, University of Colorado, Boulder, CO \\ 80309-0593, USA \\ email: Alexander.Brown@colorado.edu, Barton.Tofany@colorado.edu, \\ Thomas.Ayres@@colorado.edu \\ ${ }^{2}$ European Southern Observatoty, Garching, Germany hkorhon@eso.org \\ ${ }^{3}$ KIS, Freiburg, Germany sveta@kis.uni-freiburg.de \\ ${ }^{4}$ University of Washington, Seattle, WA, USA kowalski@astro.washington.edu, \\ slh@astro. washington. edu \\ ${ }^{5}$ Trinity College Dublin, Dublin, Ireland graham.harper@tcd.ie \\ ${ }^{6}$ Dept. of Astronomy \& Space Physics, Uppsala University, \\ Box 515, SE-75120 Uppsala, Sweden \\ email: piskunov@astro.uu.se
}

\begin{abstract}
The Kepler satellite provides a unique opportunity to study the detailed optical photometric variability of late-type stars with unprecedentedly long (several year) continuous monitoring and sensitivity to very small-scale variations. We are studying a sample of over two hundred cool (mid-A - late-K spectral type) stars using Kepler long-cadence (30 minute sampling) observations. These stars show a remarkable range of photometric variability, but in this paper we concentrate on rotational modulation due to starspots and flaring. Modulation at the $0.1 \%$ level is readily discernable. We highlight the rapid timescales of starspot evolution seen on solar-like stars with rotational periods between 2 and 7 days.
\end{abstract}

Keywords. Stars:spots, stars:activity, stars:rotation, stars: late-type

\section{Introduction}

Starspots are the most blatant manifestations of photospheric magnetic activity patterns on late-type stars in the optical. Starspots are dark, and thus conspicuous, because the intense magnetic fields within them suppress the convective heat flux locally, thereby cooling the atmosphere above the spot. The optical photometric monitoring provided by the Kepler satellite (Koch et al. 2010) allows study of activity phenomena such as the growth, migration, and decay of starspots, differential rotation, activity cycles, and flaring. Better understanding of how magnetic activity and dynamos work on other stars should lead to a better understanding of and predictive capability for solar activity. Individual starspots have lifetimes ranging from a few months in tidally-locked binaries to a few weeks in single active dwarfs (Hussain 2002). On active stars multiple, independent activity cycles are seen with typical shorter periods of 3-4 years and longer periods of 810 years (Olah et al. 2009). Such rotational and activity cycle periods and spot lifetimes 


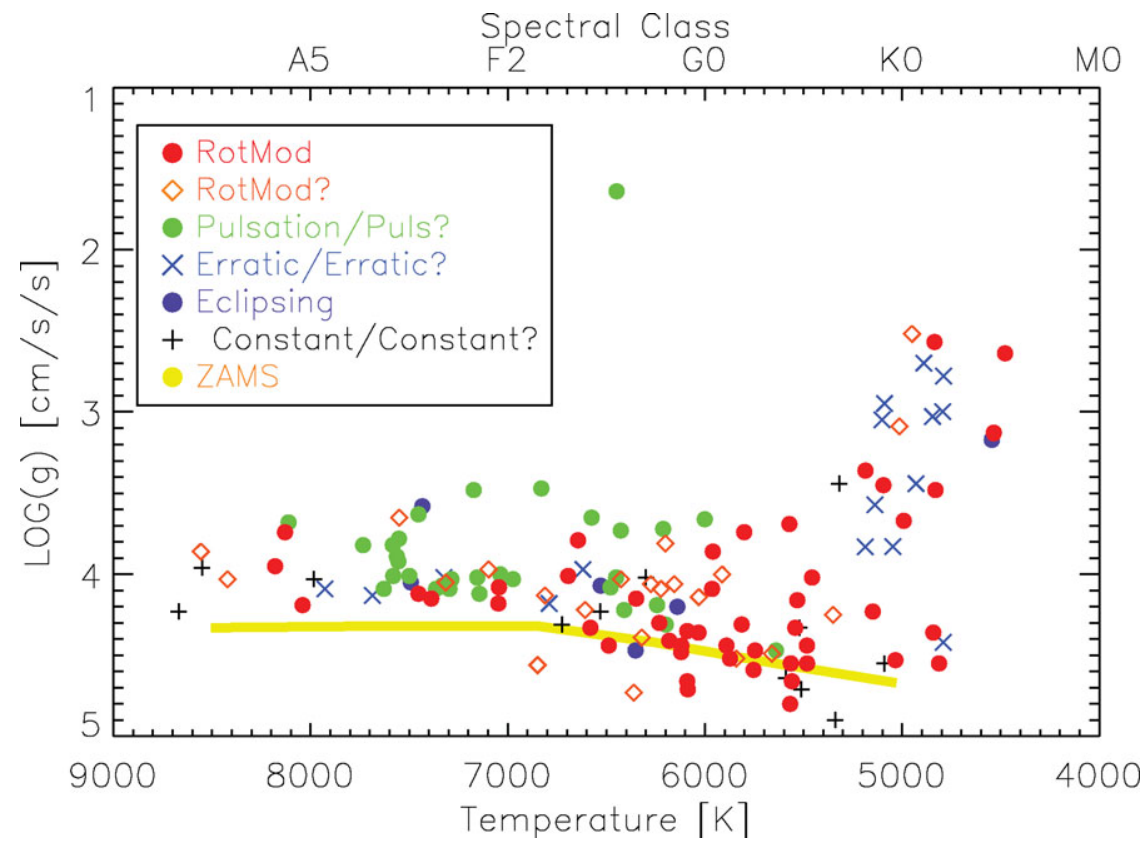

Figure 1. HR diagram for our Kepler Cycle 1 sample showing the dominant form of variability for each of these 135 stars. The effective temperatures and surface gravities are taken from the Kepler Input Catalog.

are well suited to study with the near-continuous Kepler photometry, particularly if long time series spanning the full mission lifetime $(\geqslant 3.5$ years) can be obtained.

Three key observational attributes of the solar dynamo are: (1) its cyclic behavior, showing an ebb and flow of the spotted area over an $\sim 11$-year time-scale; (2) migration of spots from mid-latitudes at the cycle onset to lower latitudes at the end (the "butterfly diagram"); and (3) preferred stable longitudes for spot emergence ("active longitudes"). Active stars, such as fast-rotating young dwarf stars and tidally-locked close binaries, clearly show enhanced magnetic activity compared with older, more slowly rotating dwarfs like the Sun. Their higher activity levels are manifested in intense X-ray, FUV, and $\mathrm{Ca}$ II $\mathrm{H}+\mathrm{K}$ emissions, and a greater surface area coverage of spots (tens of \%) than ever seen on the Sun (where sunspots typically cover only $0.01 \%$ of the surface). However, active star dynamos appear to differ substantially from the solar example: with cyclic behavior occurring on shorter time-scales and a strong tendency to show large spots at high latitudes, although still displaying preferences for active longitudes.

\section{Target Selection Using GALEX FUV and NUV Imaging}

Kepler's Field of View (FOV) covers 105 square degrees and is continuously pointed at the same area of sky in Cygnus/Lyra. However, while millions of potential stellar targets are available in the appropriate magnitude range $(\mathrm{V}=9-15)$, only data for $\sim 150,000$ stars is recorded because of telemetry limitations. Consequently, we developed a method using GALEX UV imaging to preselect stars likely to show high levels of stellar magnetic activity so that we could address our science goals of observing starspot evolution, activity patterns, differential rotation, and activity cycles on late-type stars. GALEX with a 1 

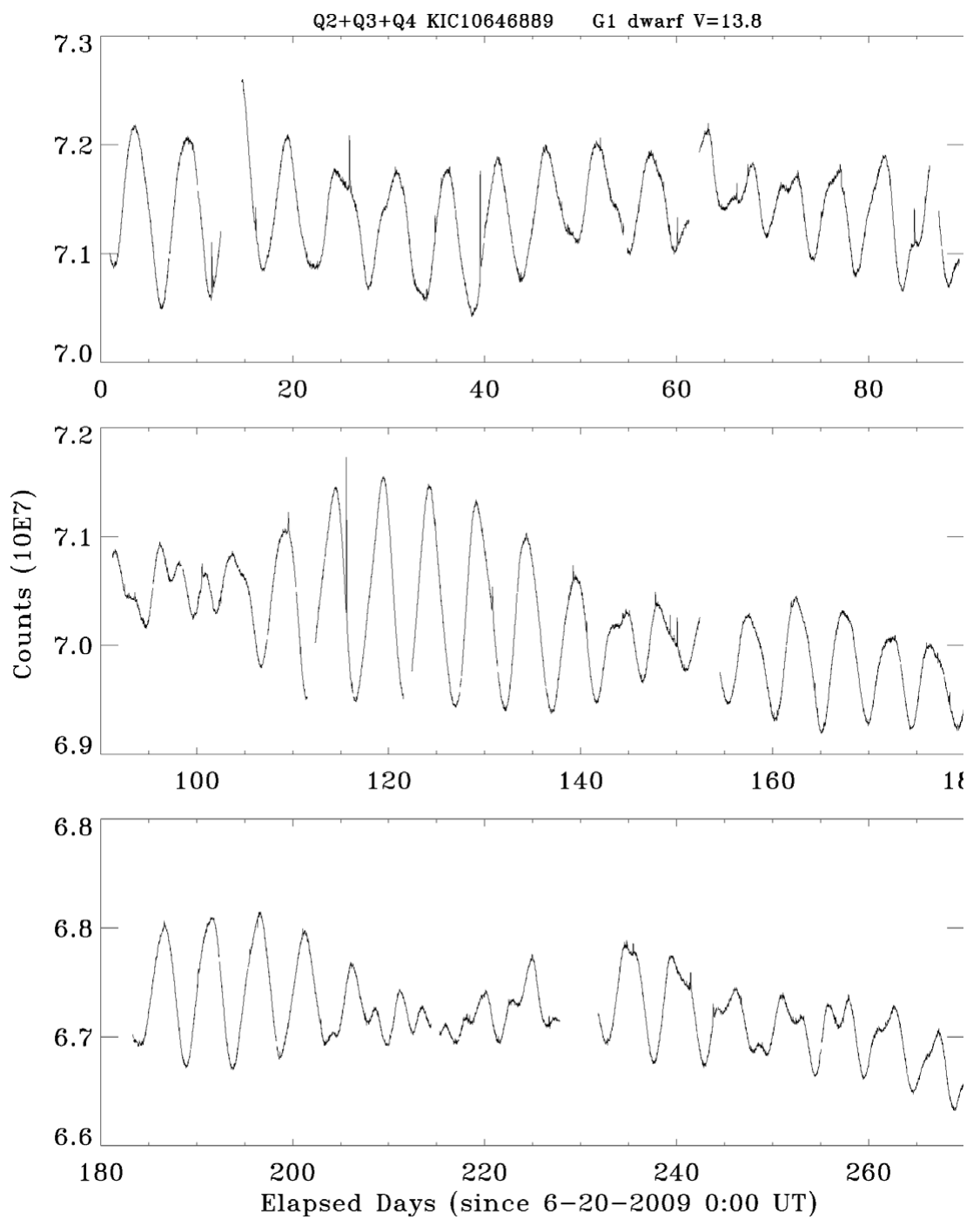

Figure 2. Kepler light-curve of the G1 dwarf KIC 10646889 over the 270 period covered by Kepler Quarters 2-3-4. Data for each Quarter is recorded by a different CCD. Prior to Kepler such time series were impossible.

square degree FOV is the only UV or X-ray satellite capable of surveying a significant fraction of the Kepler Field. Stellar activity is most obvious in the UV and X-ray spectral regions where the stellar photospheric radiation is weak. During GALEX Cycles 4 and 5 we imaged 28 square degrees of the Kepler Field using deep (up to 6 ksec) exposures. We used the FUV (1350-1800 $\AA$ ) minus NUV (1800-2800 $\AA$ ) color as a function of B-V to select active stars due to their FUV excess. We calibrated this method using nearly active and inactive stars. For inclusion in our Kepler sample stars had to - fall in our colorcolor selection box; be $3 \sigma$ sources in both GALEX channels; have a stellar counterpart in the SDSS, 2MASS, or HST GSC catalogs; have no optical companion within 10 arcsec; have $\mathrm{V}=9.0-15.0$; and lie on the active Kepler CCD area. 


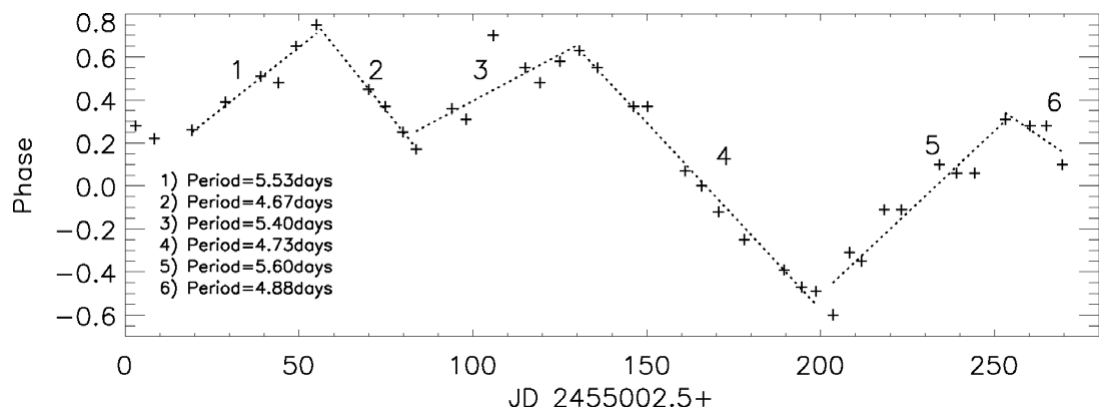

Figure 3. The early-G dwarf KIC 10646889 shows rapid spot migration in both longitude and latitude implying strong differential rotation. Alternatively, the active region "flip-flop" process might also be influencing the light-curve.

\section{Kepler Observations}

Kepler is a NASA satellite launched in March 2009 into an Earth-trailing orbit for thermal stability. It has a mission lifetime of at least 3.5 years and a primary science goal of detecting Earth-like planets through transit observations. Kepler has a vast array of 42 broadband optical (4300-8900 $\AA$ ) CCDs that are 0.95 meters across at the focal plane of a $1.4 \mathrm{~m}$ telescope. Quarterly $90^{\circ}$ rolls of the spacecraft maintain power to the spacecraft and aid thermal stability. Data downloads occur once a month.

Our GO Cycle 1 and 2 programs are obtaining 30 minute cadence photometry for a total of just over 200 stars with spectral types from mid-A to mid-K. We have currently received 9 months [3 quarters] of Kepler photometry for our Cycle 1 sample and data acquisition is on-going for our 185 Cycle 2 targets. Unprecedented near-continuous time series should result with durations of several years. An H-R diagram outlining the dominant types of variability is shown in Fig. 1.

In Fig. 2 we show 9 months of Kepler photometry for the G1 dwarf KIC 10646889, which has a mean rotational period is 5.16 days, which is rapid rotation for a solarmass star. A strongly varying modulation with a maximum amplitude of $3 \%$ is seen due to starspots migrating relative to each other. Strong evidence for differential rotation is present (as illustrated in Fig. 3 where the apparent rotation period is measured for week-long subsamples of the data) - as dominant spots move to higher latitudes, longer apparent rotation periods are measured.

\section{Conclusions}

The Kepler data are of high quality and allow study of low amplitude variability. A wide diversity of types of variability is seen — including rotational modulation, pulsation, eclipses, flares, and erratic variability. Roughly one third of the stars shows strong rotational modulation, while a further third are dominated by pulsation. Pulsation is generally restricted to earlier A-F spectral types. White-light flares are common on rapidlyrotating solar-like stars. Erratic variability is typical for cooler giant stars. Kepler provides a unique, near-continuous record of stellar optical variability that allows detailed study of starspot modulation and the growth, decay and migration of starspots for stars with a wide range of fundamental properties. Rotational modulation periods range from a $\mathrm{K}$ dwarf with a 0.6 day period to a $\mathrm{K}$ giant with a 40 day period. The light-curve of the G1 dwarf KIC 10646889 illustrates the dramatic spot migration and strong inferred differential rotation that can be observed. 


\section{Acknowledgments}

This work was supported by NASA Kepler grant NNX10AC51G and GALEX NNX08AU63G and NNX09AM47G to the University of Colorado.

\section{References}

Hussain, G. 2002, Astron. Nachr., 323, 349

Koch, D. G., et al., 2010, Astrophys. J. Lett., 713, L79

Olah, K., et al., 2009, Astron. Astrophys, 501, 703 